\title{
Determination of the single-phase constitutive relations of $\alpha / \beta$ dual phase TC6 titanium alloy
}

\author{
Ran Shi ${ }^{\mathrm{a}, \mathrm{b}}$, Guoju Li ${ }^{\mathrm{a}, \mathrm{b}}$, Zhihua Nie ${ }^{\mathrm{a}}$, Qunbo Fan ${ }^{\mathrm{a}, \mathrm{b}, *}$ \\ ${ }^{a}$ School of Materials Science and Engineering, Beijing Institute of Technology, Beijing \\ 100081, China \\ ${ }^{b}$ National Key Laboratory of Science and Technology on Materials Under Shock and \\ Impact, Beijing 100081, China \\ * Corresponding author: Email: fanqunbo@ bit.edu.cn, Tel: 8610-68911144-863, Fax: \\ 8610-68911144-866
}

\begin{abstract}
The constitutive relations of $\alpha$ and $\beta$ phases in a TC6 titanium alloy were determined by implementing a two-phase elastic-plastic self-consistent (EPSC) framework combined with the evolution of lattice strains; these strains were obtained via in-situ tensile loading synchrotron-based x-ray diffraction experiments. It was found that the $\{200\}_{\beta}$ reflection has the lowest stiffness and load partitions prior to the $\alpha$ phase during the elastic loading stage in this alloy. The simulated parameters including the diffraction elastic constant and initial yield stress of lattice reflections exhibited satisfactory correspondence with the experimental results. Further analysis of the characteristics of the Schmid Factor (SF) distributions of the main slip systems revealed that the elastic-plastic transition process in the $\alpha$ phase occurs over a prolonged period. In contrast, the $\beta$ phase undergoes a transient process owing to its relatively more concentrated SF frequency distributions, than those of the $\alpha$ phase. In addition, the fitted stress-strain curve of each phase was compared with
\end{abstract}


the measured macro stress-strain curve obtained from the in-situ experiment. It revealed Young's moduli of $110.3 \mathrm{GPa}$ and $104.5 \mathrm{GPa}$, and yield stresses of $877.8 \mathrm{MPa}$ and 969 $\mathrm{MPa}$, for the $\alpha$ and $\beta$ phases, respectively.

Keywords: Synchrotron x-ray diffraction; EBSD; Titanium alloys; Constitutive relation; Schmid Factor

\section{Introduction}

Recently, the high-performance $\alpha / \beta$ dual phase TC6

(Ti-6Al-2.5Mo-1.5Cr-0.5Fe-0.3Si) titanium alloy has been commonly used in aerospace industries for fabrication of some of the critical engine components. This alloy has outstanding mechanical properties, such as high strength-to-weight ratio and fatigue resistance, as well as excellent ductility and toughness. To develop micromechanical models for investigating the macroscopic and microscopic behavior of multi-phase materials, such as this alloy, the constitutive relation of each constituent phase must be determined. Each phase in a multi-phase material is usually obtained via heat treatments, based on TTT plots or phase diagrams, or by using a possibly substitute material, which has almost the same composition [1-3]. However, the chemical composition has a significant effect on the structure and mechanical properties of the phase, and hence the single-phase behavior differed from that of the phases in the aggregate material. Ramazani et al. [4] and Hosseini-Toudeshky et al. [5] evaluated the stress-strain behaviors of the constituent phases in dual-phase steel by using classical dislocation theory. These empirical theories were, however, based on experiments performed over a 
limited range of chemical composition and manufacturing processes, and hence their validity remains questionable [1]. Recently, Ghassemi-Armaki et al. determined the deformation response of ferrite and martensite by performing nanoindentation and uniaxial compression on focused ion beam-milled cylindrical micropillars [6]. However, the size effect of both the indentation and the micropillars limit their practical applications $[7,8]$.

In-situ synchrotron-based high-energy X-ray diffraction (HEXRD) is a non-destructive strongly penetrative method that can be used to analyze microstresses in a material. In fact, in-situ diffraction experiments, during tensile loading, can be combined with mathematical tools that can replace the commonly used empirical or phenomenological laws, to acquire data that cannot be directly measured during experiments. The elastic-plastic self-consistent (EPSC) framework provides an intermediate view into the microscopic behaviors, and excellent agreement with diffraction data has been obtained for various materials, with differing crystal structures, and multiphase systems in composites [9-13]. The EPSC framework, and extensions thereof, are computationally efficient. Furthermore, this framework has proven effective for predicting the macroscopic stress-strain response and evolution of lattice strains, residual strains, relative activity of the slip and twinning deformation modes, and the development of crystallographic texture [14-17]. In this study, a two-phase version of the framework is used to optimize the intrinsic constants of the TC6 titanium alloy as well as obtain the lattice strain response and constitutive relations of each phase. 
In addition, microstructural features, such as the grain size distribution and volume fraction of each phase, of the alloy were determined via electron backscattered diffraction (EBSD) measurements. Then the EPSC framework, used in this study, is briefly described. The initial orientation of the grains was obtained from EBSD measurements. Based on the results of in-situ synchrotron radiation experiments and SF distributions of the main slip systems in each phase, the parameters in the framework are adjusted to optimum values. The phase constitutive relations of each phase and parameters of the micro slip systems were subsequently obtained. Moreover, it is shown that the elastic-plastic transition modes of the $\alpha$ and $\beta$ phases are different.

\section{Experimental description}

\subsection{In-situ synchrotron-based HEXRD experiments}

In-situ synchrotron-based HEXRD experiments were performed on the beamline 11-ID-C at Advanced Photon Source (APS) at Argonne National Laboratory. The energy of the monochromatic X-ray beam is $111 \mathrm{keV}$ (with wavelength of $0.11165 \AA$ ). The experimental setup is shown in Fig. 1. The dog-bone-shaped sheet specimen of forged TC6 titanium alloy with gauge section dimensions of $3.175 \mathrm{~mm} \times 10.16 \mathrm{~mm} \times 1 \mathrm{~mm}$ was mounted to a loading frame. $\mathrm{CeO}_{2}$ powder was used as standard sample for calibrating the tilt angles and the distance between the specimen and a two-dimensional (2D) detector which was used to collect the diffraction patterns with sampling times of $\sim 1 \mathrm{~s}$

during tensile loading. The images of the Debye rings were segmented using the software 
program Fit2D [18] and plots of diffraction angle $2 \theta$ against intensity were integrated from $10^{\circ}$ regions of diffraction planes normal to the longitudinal loading direction (LD). The Bragg angle $\theta_{h k l}$ of each $h k l$ plane was determined by fitting the individual peak or overlapped peaks in the diffraction spectra by employing two Gaussian functions with different peak widths.

In this case, for each $h k l$ plane, the elastic lattice strain $\varepsilon_{h k l}$ can be calculated from the measured lattice spacing $d_{h k l}$ and a reference lattice spacing $d_{0, h k l}$, as

$\varepsilon_{h k l}=\frac{d_{h k l}-d_{0, h k l}}{d_{0, h k l}}$

where $d_{0, h k l}$ is the interplanar spacing of the specified $h k l$ plane during the initial elastic load at zero.

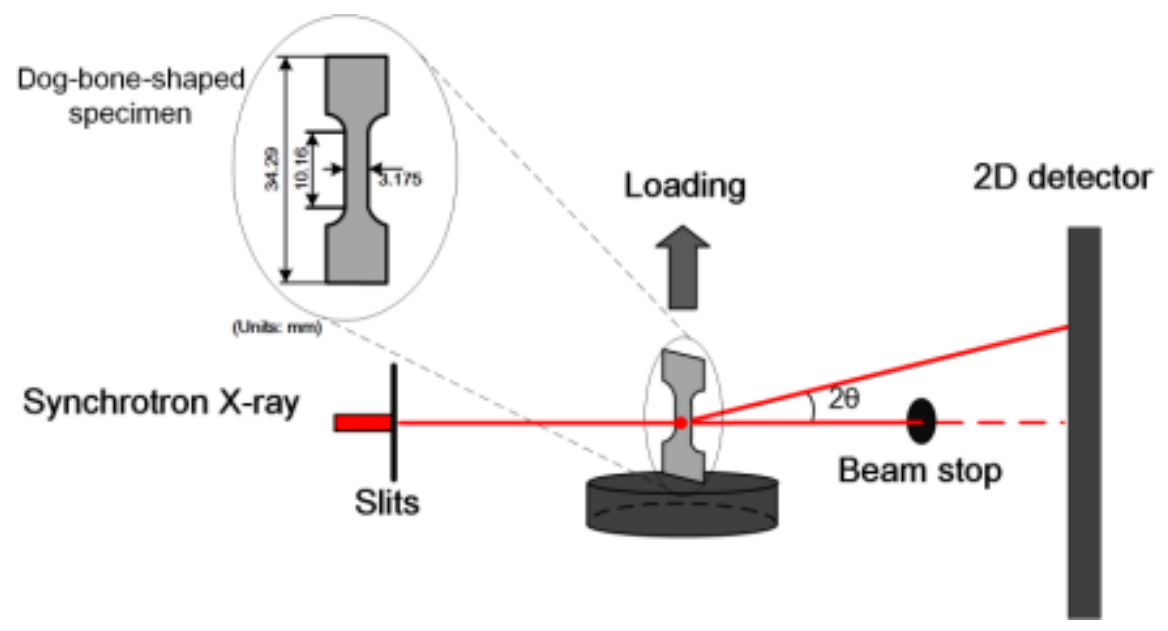

Fig. 1. Schematic of the experimental set-up for in-situ loading studies by HEXRD. 2.2 Materials and EBSD experiments 
The as-received TC6 titanium alloy (Baoji Nonferrous Metal Works forged product, Baoji, China) (also known as BT3-1 [19, 20]) with the chemical composition listed in Table 1 was forged and annealed at $800{ }^{\circ} \mathrm{C}$ for $2 \mathrm{~h}$ followed by air cooling. Prior to in-situ deformation tests, EBSD analyses were performed on the longitudinal surface of the alloy. The dog-bone-shaped sheet specimen was prepared by wire-electrode cutting along the axis direction of the forged bar. The surface of the specimen was mechanically ground and polished, because the samples designated for EBSD measurement must have a flat highly polished (mirror-finish) surface. Furthermore, removal of the residual stress, owing to mechanical polishing, from the surface layers is essential to obtain a satisfactory pattern indexing rate. This stress was removed via electropolishing at a voltage of $25 \mathrm{~V}$, for $\sim 30 \mathrm{~s}$, in a solution of $6 \%$ perchloric acid $\left(\mathrm{HClO}_{4}\right), 34 \%$ butan-1-ol $\left(\mathrm{CH}_{3}\left(\mathrm{CH}_{2}\right)_{3} \mathrm{OH}\right)$, and $60 \%$ methanol $\left(\mathrm{CH}_{3} \mathrm{OH}\right)$ at $25^{\circ} \mathrm{C}$.

Table 1. Chemical composition of TC6 experimental alloy (wt\%).

\begin{tabular}{llllllllll}
\hline Element & $\mathrm{Al}$ & $\mathrm{Mo}$ & $\mathrm{Cr}$ & $\mathrm{Fe}$ & $\mathrm{Si}$ & $\mathrm{C}$ & $\mathrm{N}$ & $\mathrm{H}$ & $\mathrm{Ti}$ \\
\hline wt\% & 6 & 2.5 & 1.5 & 0.5 & 0.3 & $<0.07$ & $<0.03$ & $<0.01$ & Bal. \\
\hline
\end{tabular}

In our study, the EBSD experiment was carried out by using FEI Nanosem 430 field emmission gun scanning electron microscope (SEM, HITACHI S-4800N, Japan) operated at $20 \mathrm{kV}$. Several $400 \times 400 \mu \mathrm{m}^{2}$ EBSD maps, collected at a step size of $0.65 \mu \mathrm{m}$, were stitched together and analyzed using the Oxford HKL Channel 5 software package. Fig. 2 shows the grain size maps and histograms of the $\alpha$ and $\beta$ phases. Grain boundaries 
were defined as having $>15^{\circ}$ misorientation. As the figure shows, smaller grains are shaded in darker color than larger grains. The statistical analysis revealed average grain sizes of $2.63 \mu \mathrm{m}$ and $1.94 \mu \mathrm{m}$ for the $\alpha$ and $\beta$ phases, respectively.
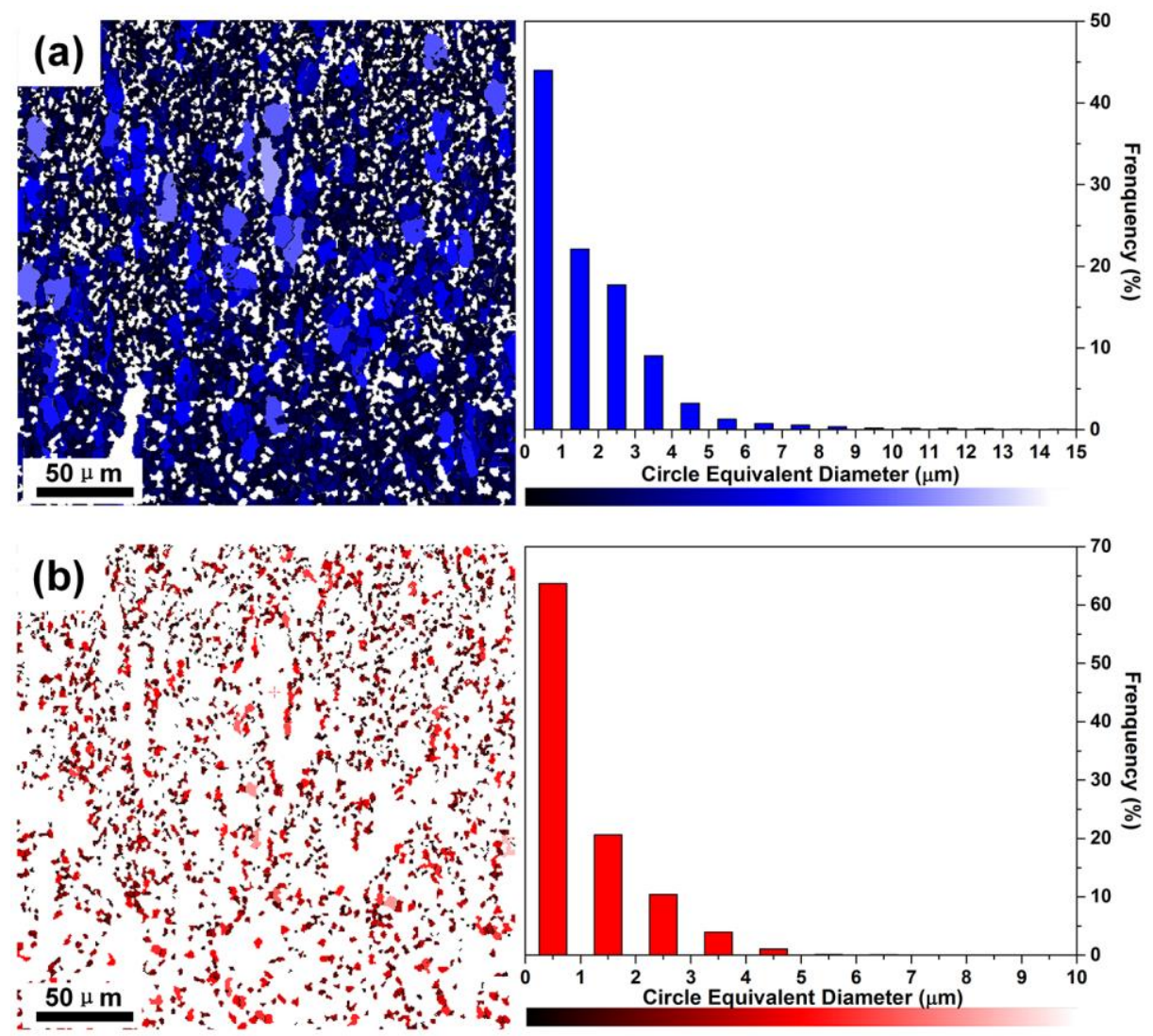

Fig. 2. Grain size map and histogram of the (a) $\alpha$ phase and (b) $\beta$ phase.

The microstructure is accurately reproduced in the EBSD phase map (see Fig. 3), which reveals the distribution of the body-center cubic (BCC)- $\beta$ phase (red) and the hexagonal-close-packed (HCP)- $\alpha$ phase (blue). The phase constitute volume fractions are $w(\alpha)=81.8 \%$ and $w(\beta)=18.2 \%$. 


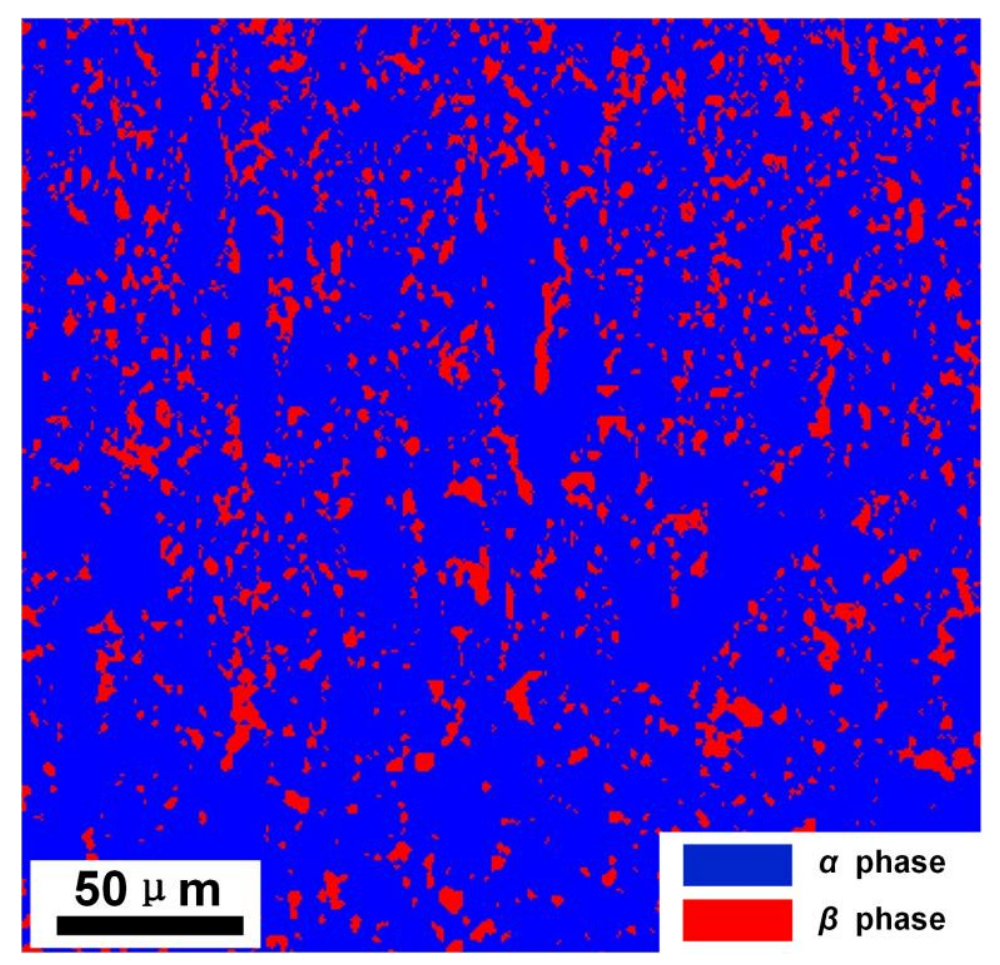

Fig. 3. Phase distribution map of the $\mathrm{HCP}-\alpha$ phase (blue) and the $\mathrm{BCC}-\beta$ phase (red).

\section{Determining the constitutive relations}

\subsection{Single-phase EPSC framework}

The EPSC framework plays a significant role in establishing the correlation between the experimental data and the simulation results, and provides a preferable approach for obtaining the constitutive relation for each phase. The elastic-plastic behavior of a polycrystalline aggregate has been described by using a modification of the Hill self-consistent model [21], which was first implemented by Hutchinson [22]. An underlying assumption of this model is that the aggregate is composed of single crystals, and the corresponding distribution of orientations and volume fractions match the measured texture. Each grain is taken to be an elastically anisotropic, ellipsoidal inclusion embedded in an infinite homogeneous medium that represents the rest of the aggregate [23]. As such, anisotropic elastic constants and slip mechanisms of a single crystal, of the respective phase, are assigned to each grain. Interactions between individual grains and 
the surrounding medium are performed by using an elastic-plastic Eshelby-type self-consistent formulation [24]. As Eshelby observed, the stress and strain rates in the ellipsoidal inclusion are uniform and can be related to the stress and strain rates at infinity by fourth order tensors $A_{\mathrm{c}}$ and $B_{\mathrm{c}}$ according to:

$\dot{\varepsilon}_{\mathrm{c}}=A_{\mathrm{c}} \dot{\bar{\varepsilon}}$ and $\dot{\sigma}_{\mathrm{c}}=B_{\mathrm{c}} \dot{\bar{\sigma}}$

and, where consistent with the Hooke's law which is extended to the elastic-plastic regime by expressing it as a rate and introducing an instantaneous moduli $L$ relating the macroscopic stress and strain increment:

$\dot{\bar{\sigma}}=L \dot{\bar{\varepsilon}}$ and $\dot{\bar{\varepsilon}}=M \dot{\bar{\sigma}}$

where $M=L^{-1}$ except when the inverse fails to exist. By extension, for each ellipsoidal grain is:

$\dot{\bar{\sigma}}_{\mathrm{c}}=L_{\mathrm{c}} \dot{\bar{\varepsilon}}_{\mathrm{c}}$ and $\dot{\bar{\varepsilon}}_{\mathrm{c}}=M_{\mathrm{c}} \dot{\bar{\sigma}}_{\mathrm{c}}$

Hill [21] showed that the calculation of $A_{\mathrm{c}}$ and $B_{\mathrm{c}}$ is facilitated by introducing a “constraint" tensor $L^{*}$ with inverse $M^{*}$ that:

$A_{\mathrm{c}}=\left(L^{*}+L_{\mathrm{c}}\right)^{-1}\left(L^{*}+L\right)$ and $B_{\mathrm{c}}=\left(M^{*}+M_{\mathrm{c}}\right)^{-1}\left(M^{*}+M\right)$

where $L^{*}$ is the effective stiffness, related to the Eshelby tensor $S$ by:

$L^{*} S=L(I-S)$

where $I$ is the fourth order identity tensor. The weighted averages of stress and strain rates over all the grain orientations of the polycrystalline are equal to the overall stress and strain (the average over all the grains is denoted by \{\} ):

$\left\{\dot{\sigma}_{\mathrm{c}}\right\}=\dot{\bar{\sigma}}$ and $\left\{\dot{\varepsilon}_{\mathrm{c}}\right\}=\dot{\bar{\varepsilon}}$

Finally, combining Eqs. (2)-(4), an expression for the overall self-consistent moduli can be yielded:

$L=\left\{L_{\mathrm{c}} A_{\mathrm{c}}\right\}$ and $M=\left\{M_{\mathrm{c}} B_{\mathrm{c}}\right\}$ 
The single-crystal elastic constants and the various deformation systems activated at predetermined values of the critical resolved shear stress (CRSS, $\left.\tau_{0}\right)$ describe the elastic response of the individual grains and the plastic response, respectively. Furthermore, hardening on each deformation system is described by the extended Vocé hardening law, which is given as follows [25]:

$$
\tau(\Gamma)=\tau_{0}+\left(\tau_{1}+\theta_{1} \Gamma\right)\left[1-\exp \left(\frac{-\theta_{0} \Gamma}{\tau_{1}}\right)\right]
$$

where $\tau_{0}, \theta_{0}$, and $\Gamma$ are the initial critical resolved shear stress, initial hardening rate, and accumulated shear strain on the slip system, respectively. The final hardening parameter is denoted as $\theta_{1}$, and $\left(\tau_{0+} \tau_{1}\right)$ is the intercept of the slope at $\Gamma=0$. The parameters in Eq. (9) are derived by fitting the in-situ experimental true stress-strain curve of the material (as shown in Fig. 8).

Based on their activities, the various slip systems harden each other. The increment of the CRSS, $\dot{\tau}^{i}$, is related to the shear rate of the $j^{\text {th }}$ slip system, $\dot{\gamma}^{j}$, by:

$$
\dot{\tau}^{i}=\frac{d \tau^{i}(\Gamma)}{d \Gamma} \sum_{j} h^{i j} \dot{\gamma}^{j}
$$

where $h^{i j}$ are the hardening coefficients relating the hardening of each system $i$ to the other system $j$ and itself.

\subsection{Two-phase framework}


To implement the above theory to two-phase polycrystalline materials, the framework of single-phase EPSC remains the same, however, the overall instantaneous moduli $\dot{L}$ now depends upon the properties of both phases as a linear combination of the equivalent instantaneous moduli of each phase, weighted by the volume fractions:

$\dot{L}=w(\alpha) \cdot \dot{L}_{\alpha}+w(\beta) \cdot \dot{L}_{\beta}$

The properties of each phase are calculated independently and then combined in the calculation of the overall moduli, reflecting the interaction between phases consequently [26].

\section{Discussion}

In the EPSC framework, the evolution of strain is determined for unidirectional strain loading in strain steps of $0.1 \%$. To correlate the orientation of single crystals with those on polycrystalline samples, crystallographic data are described by means of three Euler angles which are commonly used to describe the sample orientation relative to the crystal [27-29]. For orientation measurements in Channel 5, the convention of Bunge is applied. The Euler angles: $\varphi 1, \Phi, \varphi 2$ represent a rotation of $\varphi 1$ about the z-axis, followed by a rotation of $\Phi$ about the rotated $\mathrm{x}$-axis and followed by a rotation of $\varphi 2$ about the rotated z-axis [30]. Therefore, a set of discrete Euler angles of $6495 \alpha$ grains and $2341 \beta$ grains are specified and weighted, based on the EBSD orientation distribution measurement. EBSD crystalline orientation maps (Inverse pole figures, IPFs) (Fig. 4) are 
colored according to the initial crystallographic orientation of each grain in the polycrystalline material paralleling to the LD of the sample within the coordinate system comprised by three important low-index axes.
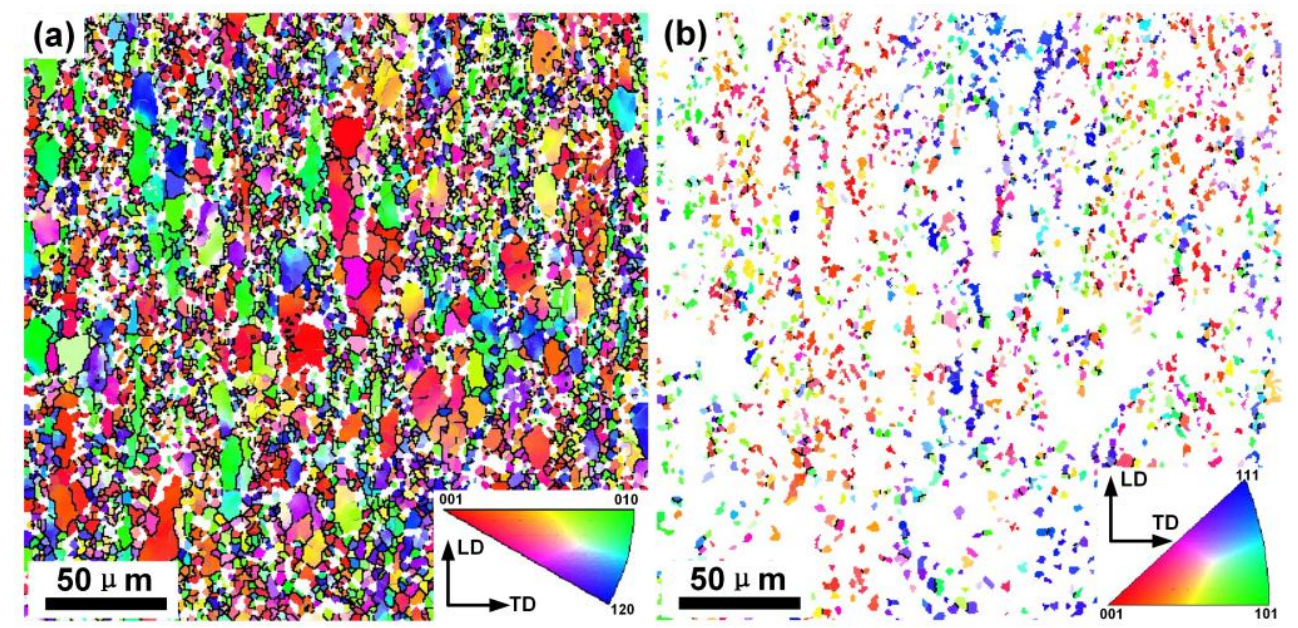

Fig. 4. Crystalline orientation maps of (a) $\alpha$ phase and (b) of $\beta$ phase.

In the EPSC framework, several material parameter inputs are required in order to account for the grain-to-grain interactions occurring within the respective phases and the elastic-plastic deformations triggered by several slip systems. These parameters include an elastic part, such as the single-crystal elastic stiffness matrix, $\boldsymbol{C}_{i j}$, and a plastic part, such as potentially available slip modes and hardening coefficients.

The initial preset $C_{i j}$ components used in the model are $C_{11}=168 \mathrm{GPa}, C_{12}=95 \mathrm{GPa}$, $C_{13}=69 \mathrm{GPa}, C_{33}=191 \mathrm{GPa}, C_{44}=48 \mathrm{GPa}$ for the $\alpha$ phase, and $C_{11}=134 \mathrm{GPa}, C_{12}=110 \mathrm{GPa}$, $C_{44}=55 \mathrm{GPa}$ for the $\beta$ phase $[31,32]$. Table 2 lists the diffraction elastic constants, $E_{h k l}$, of the lattice reflections of the $\alpha$ and $\beta$ phases, as determined from simulation, along the LD, 
by the EPSC framework; and the $E_{h k l}$ values obtained by experimentally measured lattice strains as a function of the applied stress:

$$
E_{h k l}=\frac{\sigma_{a p p l i e d}}{\varepsilon_{h k l}}
$$

Table 2. Experimental measured and EPSC simulated $E_{h k l}(\mathrm{GPa})$ of the lattice reflections of the $\alpha$ and $\beta$ phases

\begin{tabular}{cccccccc}
\hline Planes & $\{10 \overline{1} 0\}_{\alpha}$ & $\{0002\}_{\alpha}$ & $\{10 \overline{1} 1\}_{\alpha}$ & $\{10 \overline{1} 2\}_{\alpha}$ & $\{110\}_{\beta}$ & $\{200\}_{\beta}$ & $\{310\}_{\beta}$ \\
\hline$E_{h k l}^{E x p .}$ & 106.7 & 128.23 & 113.59 & 119.49 & 105.6 & 93.27 & 97.02 \\
\hline$E_{h k l}^{S C}$ & 106.25 & 137.09 & 111.62 & 123.82 & 106.18 & 93.06 & 97.39 \\
\hline
\end{tabular}

As the table shows, an excellent correspondence is obtained between the measured and simulated diffraction elastic constants. It is observed that the $\{200\}_{\beta}$ reflection has the lowest stiffness. The $\{110\}_{\beta}$ reflection, which has the largest stiffness of all those observed in the $\beta$ phase, has a stiffness lower than any reflection in the $\alpha$ phase. Therefore, the stress in the $\alpha$ phase would be higher than the $\beta$ phase indicating that load partitions prior to the $\alpha$ phase during the elastic loading stage in this alloy. The fitted components of $\boldsymbol{C}_{i j}$ of each phase are listed in Table 3.

Table 3. Fitted single-crystal elastic constants $\boldsymbol{C}_{i j}(\mathrm{GPa})$ of the $\alpha$ and $\beta$ phases 


\begin{tabular}{llllll}
\hline & $\mathrm{C}_{11}$ & $\mathrm{C}_{12}$ & $\mathrm{C}_{13}$ & $\mathrm{C}_{33}$ & $\mathrm{C}_{44}$ \\
\hline$\alpha$ phase & 168 & 97 & 77 & 198 & 43 \\
$\beta$ phase & 139.2 & 80.4 & 80.4 & 80.4 & 46 \\
\hline
\end{tabular}

EBSD mapping was also used to reveal the SF $(m)$ associated with different grain orientations [33, 34]; and this mapping revealed the grains that experienced high or low resolved shear stresses $(\tau)$ during tensile loading.

$\tau=m \cdot \sigma=\cos (\theta) \cos (\lambda) \cdot \sigma$

where $\theta$ is the angle between the direction of the applied load and the slip plane normal; $\lambda$ is the angle between the direction of the applied load and the slip direction; $\sigma$ is the applied stress or load. The CRSS is the component of critical applied stress, resolved in the direction of slip in a slip plane, required to initiate slip in a grain (plastic deformation of metals).

Fig. 5 and Fig. 6 show the SF maps and histograms of the main slip systems in the $\alpha$ grains: $\{0001\}<11 \overline{2} 0>$ basal, $\{10 \overline{1} 0\}<11 \overline{2} 0>$ prismatic, $\{10 \overline{1} 1\}<11 \overline{2} 0>$ first-order pyramidal, and $\{10 \overline{1} 1\}<11 \overline{2} 3>$ second-order pyramidal slip systems; the $\{1 \overline{1} 0\}<111>$, $\{11 \overline{2}\}<111>$, and $\{12 \overline{3}\}<111>$ constitute the main slip systems in the $\beta$ grains. The SF frequency distributions of slip systems in the $\alpha$ grains (Fig. 5) increase from hard (i.e., lowest-SF, $\mathrm{SF}=0$ ) orientations to soft (i.e., highest-SF, $\mathrm{SF}=0.5$ ) orientations. These four slip systems occur with frequencies of $61.98 \%, 55.06 \%, 84.18 \%$, and $85.52 \%$, respectively, in grains with SF values higher than 0.3. Plastic deformation occurs 
preferentially in high-SF grains. The orientations of these grains are referred to as favorably oriented components. Furthermore, with increasing deformation, the reorientation of deformed grains results in the rotation of unfavorably oriented grains to favorably oriented components. Sustainable plastic deformation of the $\alpha$ phase is initiated at a relatively large timescale. However, in contrast to those of the $\alpha$ grains, the SF frequency distributions of the slip systems in the $\beta$ grains (Fig. 6) are narrow and concentrated in the vicinity of favorable orientations. In other words, this distribution consists only of SF values higher than 0.275 . Instantaneous plastic deformation is initiated when the local stress reaches the CRSS of these high-SF systems. 


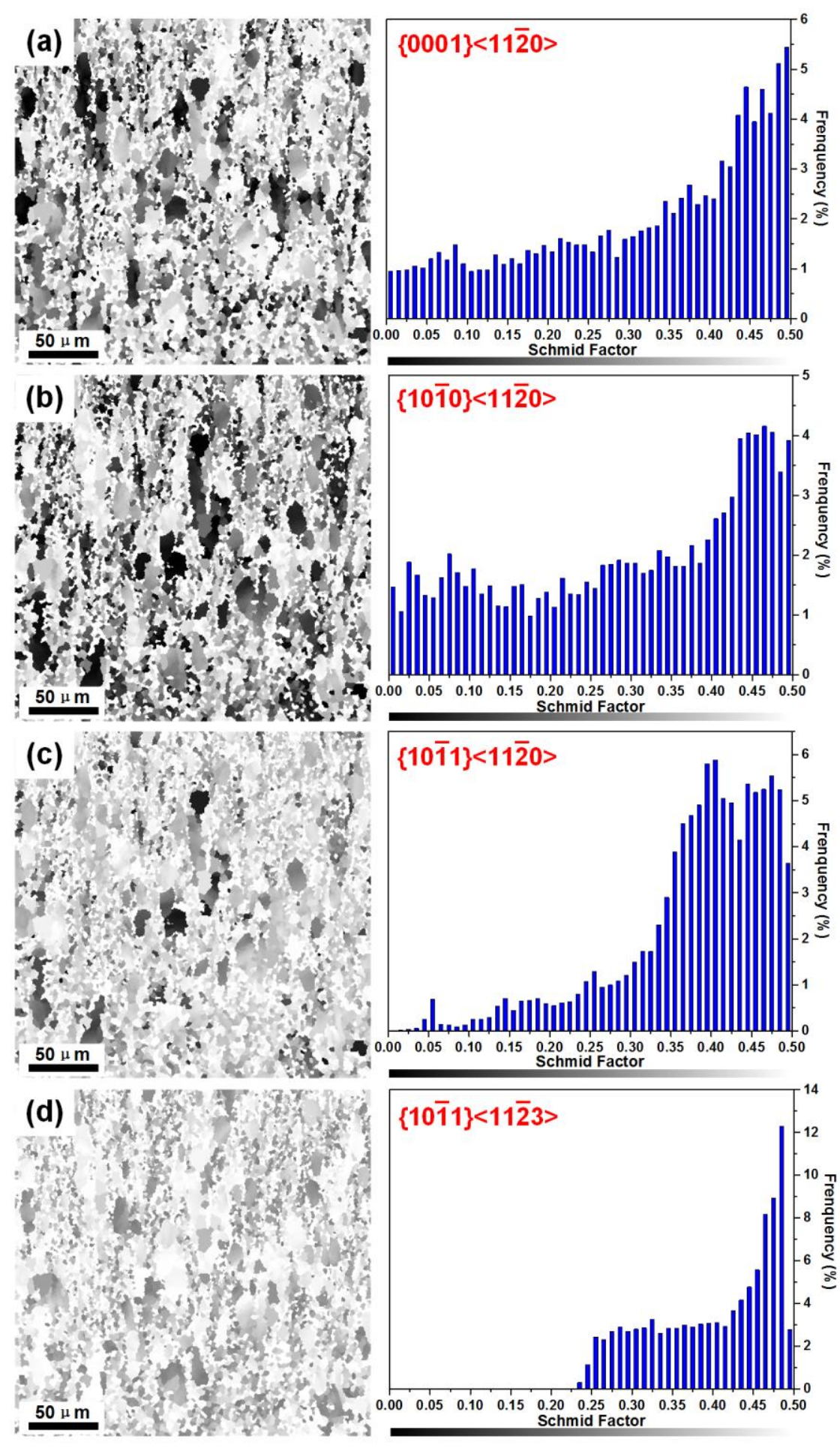

Fig. 5. Schmid factor maps and histograms of slip systems in the $\alpha$ grains: (a) $\{0001\}<11 \overline{2} 0>$ basal, (b) $\{10 \overline{1} 0\}<11 \overline{2} 0>$ prismatic, (c) $\{10 \overline{1} 1\}<11 \overline{2} 0>$ first-order pyramidal, and (d) $\{10 \overline{1} 1\}<11 \overline{2} 3>$ second-order pyramidal slip systems. 


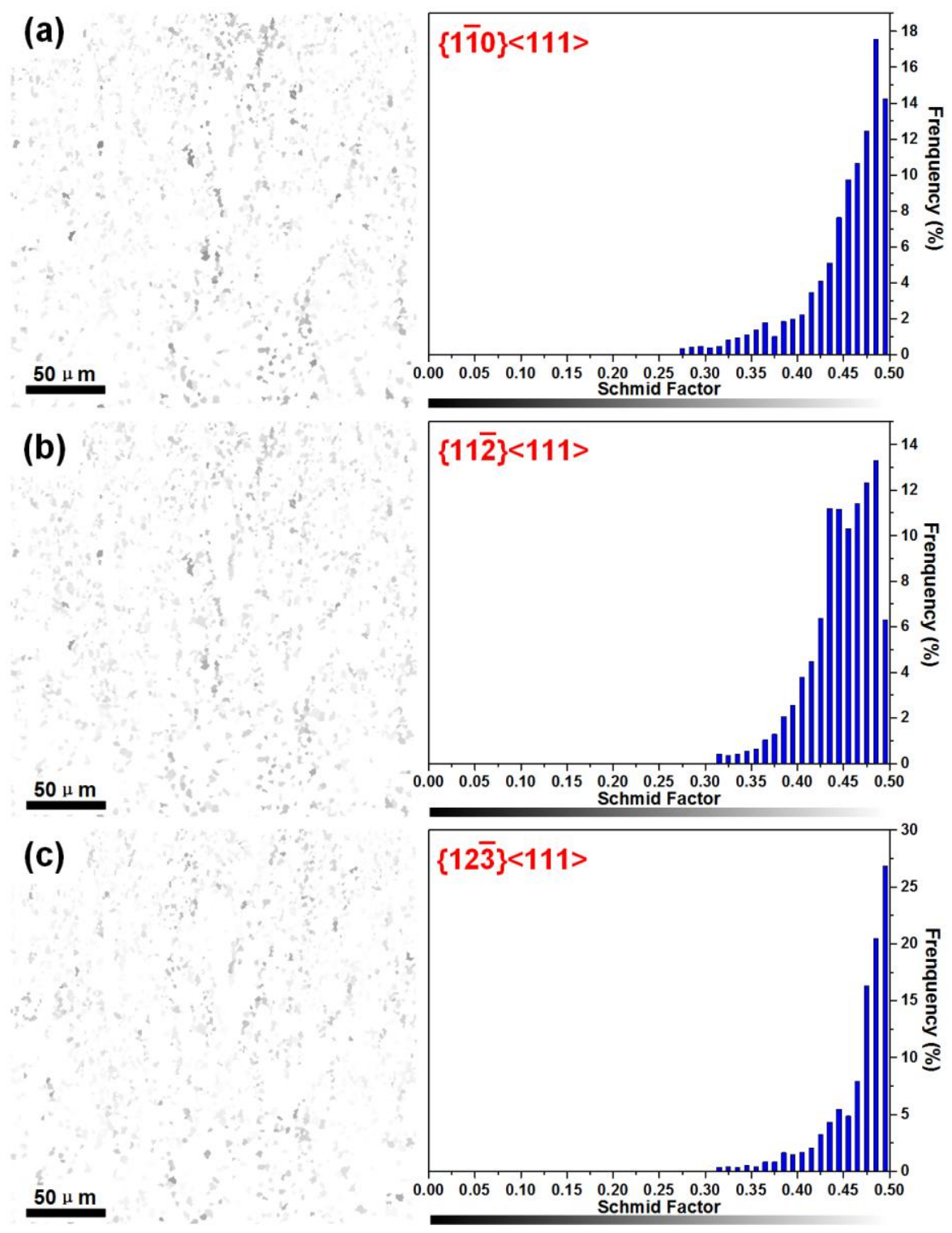

Fig. 6. Schmid factor maps and histograms of slip systems in the $\beta$ grains: (a) $\{1 \overline{1} 0\}<111>$, (b) $\{11 \overline{2}\}<111>$, and (c) $\{12 \overline{3}\}<111>$ slip systems.

As a general rule, the easiest modes to activate are those with their slip plane of greatest atomic density and the largest interplanar spacing, and with their slip direction of shortest atomic distance. It would therefore be expected that the dominant slip systems of the primary $\alpha$ phase are the basal and prismatic slip due to their relatively low CRSS [35, 
36]. Further, at room temperature, $<c+a>$ pyramidal slip mechanisms are always more difficult to activate than either prismatic or basal slip. However, they are important deformation modes, because they have much close connection with the ductility of commercial high-strength Ti alloys and have the occurrence during deformation of polycrystalline Ti alloys [37]. For $\beta$ phase, the three main slip systems shown in Fig. 6 were all employed. Thus, in the EPSC framework, the occurrence of slip deformation on the aforementioned slip systems, in each phase, was assumed.

The hardening function of the $i^{\text {th }}$ slip system of each single crystal is described by the generalized Vocé hardening law [26, 38, 39]. Fig. 7 shows the initial yield stress of each $h k l$ plane, and Table 4 lists the CRSSs and Vocé hardening parameters of each slip mode.

As shown in Fig. 7, a good agreement is achieved between experimental measurement and EPSC simulation of the initial yield stress $\left(\sigma_{s, h k l}\right)$ of lattice reflections with the deviation ranging from $1.85 \mathrm{MPa}$ for $\{0002\}_{\alpha}$ reflection to $15.58 \mathrm{MPa}$ for $\{110\}_{\beta}$ reflection.

From the fitting results, we know that, owing to their relatively low CRSSs $(0.33$ and $0.35 \mathrm{GPa}$, respectively) the basal and prismatic slip systems are the dominant slip systems of the $\alpha$ phase. The first- and second-order pyramidal slip systems have, therefore, higher critical stresses than these systems. In contrast, the plastic-deformation initiative stresses of slip systems in the $\beta$ phase are similar and relatively higher than the basal and prismatic slip systems in the $\alpha$ phase. During plastic deformation dominated by 
the sliding mechanism, the higher initial CRSS of one phase, compared to that of the other, would result in a higher yield stress.

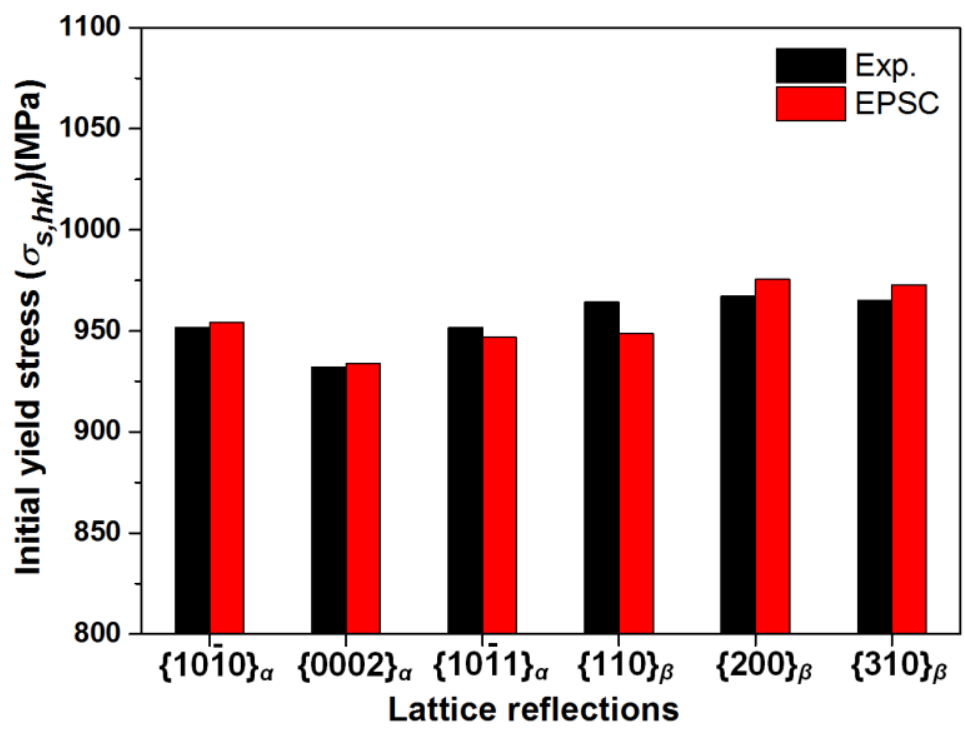

Fig. 7. Experimental measured and EPSC simulated initial yield stress $\sigma_{s, h k l}(\mathrm{MPa})$ of the lattice reflections of the $\alpha$ and $\beta$ phases.

Table 4. Critical resolved shear stresses (CRSSs) and Vocé hardening parameters (in GPa) of each slip mode used in the EPSC framework

\begin{tabular}{|c|c|c|c|c|}
\hline Slip mode & CRSS & $\tau_{1}$ & $\theta_{0}$ & $\theta_{1}$ \\
\hline$\langle 111\rangle\{110\}$ & 0.43 & & & \\
\hline$<111>\{112\}$ & 0.44 & & & \\
\hline$<111>\{123\}$ & 0.45 & & & \\
\hline$<11 \overline{2} 0>\{0002\}$ & 0.33 & 0.13 & 0.115 & 0.021 \\
\hline$<11 \overline{2} 0>\{10 \overline{1} 0\}$ & 0.35 & & & \\
\hline$<11 \overline{2} 0>\{10 \overline{1} 1\}$ & 0.47 & & & \\
\hline
\end{tabular}


The constitutive relations of $\alpha$ and $\beta$ phases simulated by EPSC are compared (Fig. 8) with the measured macro true stress-strain curve obtained from the in-situ experiment. A review of the elastic-plastic transition stage during deformation reveals that the $\alpha$ phase yields at $877.8 \mathrm{MPa}$. In contrast, the plastic deformation stage of the $\beta$ phase is initiated at a stress of $969 \mathrm{MPa}$. Additional information can be obtained from the constitutive relations. For example, the Young's moduli of the $\alpha$ and $\beta$ phases are $110.3 \mathrm{GPa}$ and 104.5 GPa, respectively.

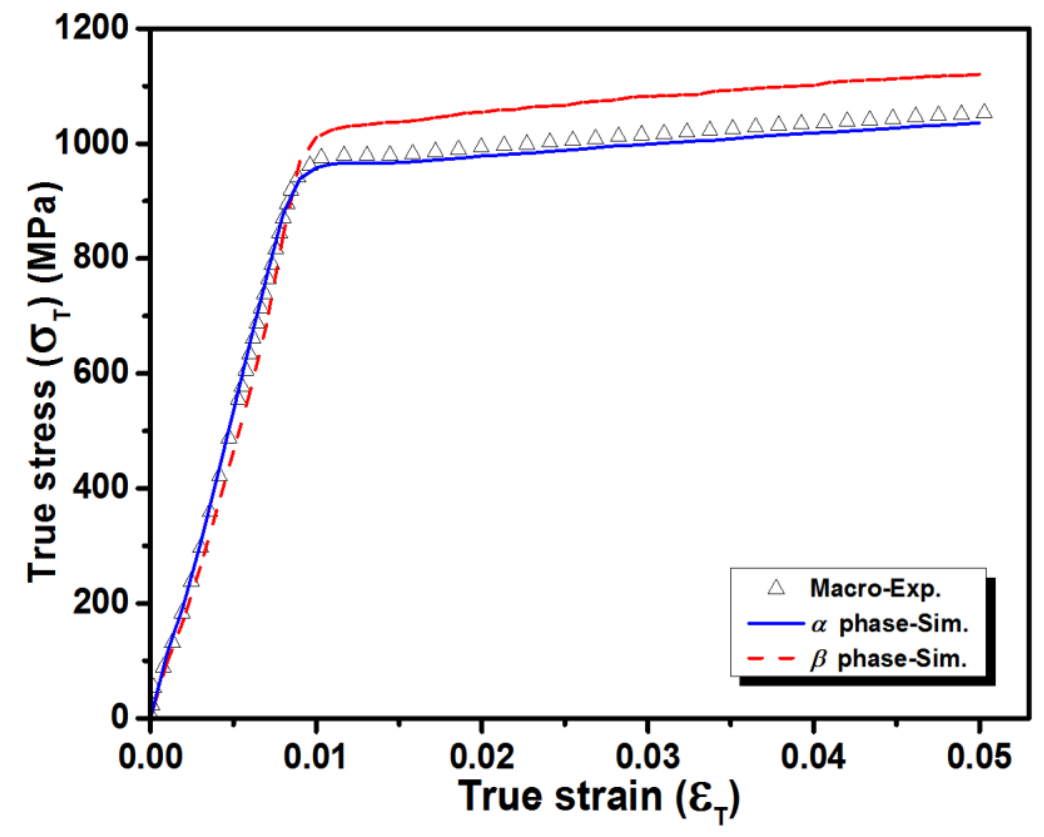

Fig. 8. The simulated constitutive relations of the $\alpha$ and $\beta$ phases, and the experimental macroscopic true stress-strain curve.

\section{Conclusions}


An EPSC framework combined with in-situ tensile loading synchrotron-based XRD was used to determine the constitutive relations of $\alpha$ and $\beta$ phases in a TC6 titanium alloy. An excellent correspondence was obtained between the experimentally determined lattice-strain evolution and the data fitted by using the two-phase framework. Moreover, characteristic parameters of the $\alpha$ and $\beta$ phases were obtained, based on the results of the framework calculation. These characteristics include the room-temperature single-crystal elastic constants, CRSSs and hardening parameters of the slip systems, Young's modulus, and the yield stresses. In the fitting framework, EBSD mapping was used to determine the initial grain orientations and to reveal the grain size distribution, phase distribution, and SF distribution of the slip systems. In addition, the SF frequency of the $\alpha$ grains increased, over a large time scale, from hard to soft orientations, whereas $\beta$ grains experienced an instantaneous elastic-plastic transition mode.

\section{Acknowledgements}

We gratefully acknowledge the funding support from project 51571031 of the Chinese National Natural Science Foundation. Use of the Advanced Photon Source was supported by the US Department of Energy, Office of Science Laboratory, Office of Basic Energy Sciences, under contract DE-AC02-06CH11357. The assistance of Dr. Yan-Dong Wang of the University of Science and Technology Beijing with the self-consistent modeling aspects is greatly appreciated.

\section{References}

[1] N. Ishikawa, D.M. Parks, S. Socrate, M. Kurihara, Micromechanical Modeling of 
Ferrite-Pearlite Steels Using Finite Element Unit Cell Models, Transactions of the Iron and Steel Institute of Japan 40(11) (2000) 1170-1179.

[2] F.M. Al-Abbasi, Predicting the deformation behavior of ferrite-pearlite steels using micro mechanical modeling of cells, Mechanics of Materials 63 (2013) 48-64.

[3] G. Ljustina, R. Larsson, M. Fagerström, A FE based machining simulation methodology accounting for cast iron microstructure, Finite Elements in Analysis and Design 80 (2014) 1-10.

[4] A. Ramazani, A. Schwedt, A. Aretz, U. Prahl, W. Bleck, Characterization and modelling of failure initiation in DP steel, Computational Materials Science 75 (2013) 35-44.

[5] H. Hosseini-Toudeshky, B. Anbarlooie, J. Kadkhodapour, Micromechanics stress-strain behavior prediction of dual phase steel considering plasticity and grain boundaries debonding, Materials \& Design 68 (2015) 167-176.

[6] H. Ghassemi-Armaki, R. Maaß, S.P. Bhat, S. Sriram, J.R. Greer, K.S. Kumar, Deformation response of ferrite and martensite in a dual-phase steel, Acta Materialia 62 (2014) 197-211.

[7] M. Delincé, P.J. Jacques, T. Pardoen, Separation of size-dependent strengthening contributions in fine-grained Dual Phase steels by nanoindentation, Acta Materialia 54(12) (2006) 3395-3404.

[8] K.E. Prasad, K. Rajesh, U. Ramamurty, Micropillar and macropillar compression responses of magnesium single crystals oriented for single slip or extension twinning, Acta Materialia 65 (2014) 316-325.

[9] B. Clausen, T. Lorentzen, M.A.M. Bourke, M.R. Daymond, Lattice strain evolution during uniaxial tensile loading of stainless steel, Materials Science and Engineering A 259(1) (1999) 17-24.

[10] M.R. Daymond, H.G. Priesmeyer, Elastoplastic deformation of ferritic steel and cementite studied by neutron diffraction and self-consistent modelling, Acta Materialia 50(6) (2002) 1613-1626.

[11] M.R. Daymond, N.W. Bonner, Lattice strain evolution in IMI 834 under applied stress, Materials Science and Engineering A 340(1-2) (2003) 272-280.

[12] M.R. Daymond, C. Hartig, H. Mecking, Interphase and intergranular stress generation in composites exhibiting plasticity in both phases, Acta Materialia 53(9) (2005) 2805-2813.

[13] M.R. Daymond, M.E. Fitzpatrick, Effect of cyclic plasticity on internal stresses in a metal matrix composite, Metallurgical and Materials Transactions A 37(6) (2006) 1977-1986.

[14] A.A. Saleh, E.V. Pereloma, B. Clausen, D.W. Brown, C.N. Tomé, A.A. Gazder, Self-consistent modelling of lattice strains during the in-situ tensile loading of twinning induced plasticity steel, Materials Science and Engineering A 589 (2014) 66-75.

[15] R. Hutanu, L. Clapham, R.B. Rogge, Intergranular strain and texture in steel Luders bands, Acta Materialia 53(12) (2005) 3517-3524. 
[16] O. Muránsky, D.G. Carr, M.R. Barnett, E.C. Oliver, P. Šittner, Investigation of deformation mechanisms involved in the plasticity of AZ31 Mg alloy: In situ neutron diffraction and EPSC modelling, Materials Science and Engineering A 496(1-2) (2008) $14-24$.

[17] M.R. Daymond, C.N. Tomé, M.A.M. Bourke, Measured and predicted intergranular strains in textured austenitic steel, Acta Materialia 48(2) (2000) 553-564. [18] A.P. Hammersley, S.O. Svensson, M. Hanfland, A.N. Fitch, D. Hausermann, Two-dimensional detector software: from real detector to idealised image or two-theta scan, High Pressure Research 14(4-6) (1996) 235-248.

[19]E. Ghasemi, A. Zarei-Hanzaki, S. Moemeni, M. Ghambari, M. Rezaee, An investigation into the warm deformation behavior of Ti-6Al-1.5Cr-2.5Mo-0.5Fe-0.3Si alloy, Materials Science and Engineering A 654 (2016) 264-270. [20] X. Xu, G. Wang, C. Xia, Stepped superplasticity deformation-induced plastic enhancement of Ti-6Al-1.5Cr-2.5Mo-0.5Fe-0.3Si alloy, Materials \& Design 36 (2012) 136-140.

[21] R.J. Hill, Continuum micro-mechanics of elastoplastic polycrystals, Journal of the Mechanics and Physics of Solids 13(2) (1965) 89-101.

[22] J.W. Hutchinson, Elastic-Plastic Behaviour of Polycrystalline Metals and Composites, Proceedings of the Royal Society of London. Series A, Mathematical and Physical Sciences 319(1537) (1970) 247-272.

[23] P.A. Turner, C.N. Tomé, A study of residual stresses in Zircaloy-2 with rod texture, Acta Metallurgica Et Materialia 42(12) (1994) 4143-4153.

[24] J.D. Eshelby, The Elastic Field Outside an Ellipsoidal Inclusion, Proceedings of the Royal Society A Mathematical Physical \& Engineering Sciences 252 (1959) 561-569.

[25] U.F. Kocks, C.N. Tomé, H.R. Wenk. Texture and anisotropy. Cambridge:

Cambridge University Press; 1998.

[26] A.M. Stapleton, S.L. Raghunathan, I. Bantounas, H.J. Stone, T.C. Lindley, D. Dye, Evolution of lattice strain in Ti-6Al-4V during tensile loading at room temperature, Acta Materialia 56 (2008) 6186-6196.

[27] U. König, B. Davepon, Microstructure of polycrystalline Ti and its microelectrochemical properties by means of electron-backscattering diffraction (EBSD), Electrochimica Acta 47 (2001) 149-160.

[28] O. Engler, V. Randle. Introduction to Texture Analysis: Macrotexture, Micro-Texture, Orientation Mapping, 2000.

[29] U.F. Kocks, C.N. Tomé, H.R. Wenk. Texture and anisotropy: preferred orientations in polycrystals and their effect on materials properties, Cambridge University press, 1998.

[30] D.J. Dingley, V. Randle, Microtexture determination by electron back-scatter diffraction, Journal of Materials Science 27 (1992) 4545-4566.

[31] B.S. Brandes, C.B. Brook. Smithells metals reference book. Oxford: Oxford University Press; 1992. 
[32] W. Petry, A. Heiming, J. Trampenau, M. Alba, C. Herzig, H.R. Schober, G. Vogl, Phonon dispersion of the bcc phase of group-IV metals. I. bcc titanium, Physical Review B Condensed Matter 43(13) (1991) 10933-10947.

[33] L. Jiang, J.J. Jonas, R.K. Mishra, A.A. Luo, A.K. Sachdev, S. Godet, Twinning and texture development in two $\mathrm{Mg}$ alloys subjected to loading along three different strain paths, Acta Materialia 55(11) (2007) 3899-3910.

[34] S. Godet, L. Jiang, A.A. Luo, J.J. Jonas, Use of Schmid factors to select extension twin variants in extruded magnesium alloy tubes, Scripta Materialia 55(11) (2006) 1055-1058.

[35] M. Zhang, F. Bridier, P. Villechaise, J. Mendez, D.L. Mcdowell, Simulation of slip band evolution in duplex Ti-6Al-4V, Acta Materialia 58 (2010) 1087-1096.

[36] F. Bridier, D.L. Mcdowell, P. Villechaise, J. Mendez, Crystal plasticity modeling of slip activity in Ti-6Al-4V under high cycle fatigue loading, International Journal of Plasticity 25 (2009) 1066-1082.

[37] J.C. Williams, R.G. Baggerly, N.E. Paton, Deformation behavior of HCP Ti-Al alloy single crystals, Metallurgical \& Materials Transactions A 33 (2002) 837-850.

[38] N. Jia, R.L. Peng, Y.D. Wang, S. Johansson, P.K. Liaw, Micromechanical behavior and texture evolution of duplex stainless steel studied by neutron diffraction and self-consistent modeling, Acta Materialia 56 (2008) 782-793.

[39] N. Jia, Z.H. Cong, X. Sun, S. Cheng, Z.H. Nie, Y. Ren, P.K. Liaw, Y.D. Wang, An in situ high-energy X-ray diffraction study of micromechanical behavior of multiple phases in advanced high-strength steels, Acta Materialia 57 (2009) 3965-3977. 